\title{
Using NMR to study fast dynamics in proteins: methods and applications
}

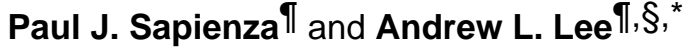 \\ "Division of Medicinal Chemistry \& Natural Products, Eshelman School of Pharmacy, University \\ of North Carolina at Chapel Hill, Chapel Hill, NC 27599, USA \\ §Department of Biochemistry and Biophysics, School of Medicine, University of North Carolina at \\ Chapel Hill, Chapel Hill, NC 27599, USA
}

\begin{abstract}
Proteins exist not as singular structures with precise coordinates, but rather, as fluctuating bodies that move rapidly through an enormous number of conformational substates. These dynamics have important implications for understanding protein function and for structure-based drug design. NMR spectroscopy is particularly well-suited to characterize dynamics of proteins and other molecules in solution at atomic resolution. Here, NMR relaxation methods for characterizing thermal motions on the picosecond-nanosecond (ps-ns) timescale are reviewed. Motion on this timescale can be conveniently captured by the Lipari-Szabo order parameter, $S^{2}$, a bond-specific measure of restriction of motion. Approaches for determining order parameters are discussed, as are recent examples from the literature that link ps-ns dynamics with conformational entropy, allostery, and protein function in general.
\end{abstract}

\section{Introduction}

As part of the drug discovery repertoire, high-resolution protein structures from x-ray crystallography and NMR spectroscopy have been invaluable for structure-based drug design. While this will continue to be the case for the foreseeable future, it is also clear that single, static models, derived from crystals bathed in cryoprotecting cosolute at $100 \mathrm{~K}$ cannot reveal all the driving forces involved in binding. Advances in drug discovery techniques depend on an improved ability to quantify "invisible forces" such as the roles of solvent, ions, and protein dynamics in the overall binding free energy change[1]*. In considering the last of these invisible forces - protein dynamics - it has been known for some time that proteins are not static entities. Rather, they are ensembles of structures, and dynamic inter-conversion between these states is important for function. NMR relaxation is one of the methods of choice for studying protein dynamics, which can, along with additional NMR experiments, interrogate processes that span timescales of picoseconds to days. While slower dynamics is clearly of interest (reviewed elsewhere[2,3]), we focus here on NMR techniques for measuring fast (picosecond-nanosecond) protein dynamics due to

(C) 2010 Elsevier Ltd. All rights reserved.

*To whom correspondence should be addressed: University of North Carolina, Division of Medicinal Chemistry \& Natural Products, Eshelman School of Pharmacy, Beard Hall, CB\# 7568, Chapel Hill, NC 27599-7568. drewlee@ unc.edu. Phone: (919)966-7821. Fax: (919) 843-5150..

Publisher's Disclaimer: This is a PDF file of an unedited manuscript that has been accepted for publication. As a service to our customers we are providing this early version of the manuscript. The manuscript will undergo copyediting, typesetting, and review of the resulting proof before it is published in its final citable form. Please note that during the production process errors may be discovered which could affect the content, and all legal disclaimers that apply to the journal pertain. 
their universality (these thermal motions are present in all proteins above the glass transition temperature), connection to conformational entropy, and implications for general function. This review aims to give the non-expert in NMR spectroscopy an introduction to the practical aspects of NMR relaxation methods for characterizing fast backbone and sidechain dynamics. Those dynamics are commonly interpreted as a single entity known as the order parameter, $S^{2}$ (not to be confused with entropy). In addition, we provide specific examples of how these tools have yielded valuable insights into biomolecular function. For more in-depth guides to both theory and implementation of backbone and side-chain relaxation experiments, readers are referred to thorough reviews by Stone[4]** and Wand[5]** respectively.

\section{Link between protein dynamics and NMR relaxation}

NMR signals (i.e. 1D, 2D, or $n \mathrm{D}$ "peaks") are generated by perturbation (through application of radiofrequency pulses) of nuclear spins away from their equilibrium states. The process by which spins return to equilibrium is termed relaxation. The energy of NMR transitions dictates that relaxation does not occur spontaneously; rather, local fluctuations of the magnetic field at specific frequencies is required [6]. Thus, there is a link between molecular dynamics and relaxation in that local magnetic field fluctuations are caused by molecular motions. Because NMR transitions are quantized, the frequencies that cause relaxation are discrete and correspond to the resonance frequencies of the nuclei involved in the transitions. For typical biomolecular nuclei $\left({ }^{15} \mathrm{~N},{ }^{13} \mathrm{C},{ }^{2} \mathrm{H}\right.$, and $\left.{ }^{1} \mathrm{H}\right)$ in a high field spectrometer (e.g. $600 \mathrm{MHz}{ }^{1} \mathrm{H}$ resonance frequency), the relaxation-promoting fluctuations occur on the ps-ns timescale.

\section{NMR relaxation methods for characterizing ps-ns dynamics of the protein backbone}

The ${ }^{1} \mathrm{H}-{ }^{15} \mathrm{~N}$ peptide amide bond vector is ideally suited for NMR relaxation studies. First, as this bond vector moves with respect to the static magnetic field due to molecular tumbling or internal motions, the local field fluctuations sensed by the ${ }^{15} \mathrm{~N}$ nucleus are dominated by the covalently attached ${ }^{1} \mathrm{H}$ dipole. Relaxation properties are therefore greatly simplified by the fact that there are no other NMR active nuclei attached. Second, relaxation measurements can be performed with variations on the simple and highly sensitive ${ }^{1} \mathrm{H}^{-15} \mathrm{~N}$ HSQC experiment. Finally, the backbone amide bond represents a probe into the flexibility of every non-proline residue in proteins.

Molecular tumbling plays a dominant role in NMR relaxation. This is why molecular size is the main influence on NMR line shapes and experimental strategies. In order to gain valuable information about the internal dynamics of proteins, these effects must somehow be de-convoluted from the effects of tumbling. Nearly thirty years ago, Lipari and Szabo proposed a method for separating local, internal motions from tumbling[7,8]. Today, this "model-free" formalism is the dominant method for interpreting dynamics data reporting on processes faster than global tumbling ( $\sim 10 \mathrm{~ns}$ for a $15 \mathrm{kD}$ protein). To characterize ps-ns dynamics from ${ }^{15} \mathrm{~N}$ relaxation, the ${ }^{15} \mathrm{~N} R_{1}$ and $R_{2}$ rates (1/T 1 and $1 / T_{2}$, respectively) in addition to the $\left\{{ }^{1} \mathrm{H}\right\}-{ }^{15} \mathrm{~N}$ heteronuclear NOE are measured[9] (Figure 1). Experiments are ideally carried out at two static magnetic field strengths. This increases the robustness of fits and allows for fitting the data to more complicated models of motion (see below). These six relaxation parameters $\left(R_{1}, R_{2}\right.$, and NOE at two fields) are then fit to model-free equations. ModelFree[10] and TENSOR2[11] are two free and downloadable fitting software packages with excellent documentation. In its basic form, there are three model-free parameters: $\tau_{\mathrm{m}}$, $S^{2}$, and $\tau_{\mathrm{e}}$. Overall tumbling of the protein is described by $\tau_{\mathrm{m}}$. Once $\tau_{\mathrm{m}}$ is initially extracted from the data, it is essentially fixed and bond-vector-specific (N-H in this case) values of $S^{2}$ 
and $\tau_{\mathrm{e}}$ that correspond to motions within the molecular frame can be fitted to the relaxation data. The $S^{2}$ "order parameter" can vary from $0-1$, with 1 corresponding to a rigid bondvector and 0 corresponding to complete flexibility. The $\tau_{\mathrm{e}}$ parameter is the characteristic time of the bond-vector motion, and typically takes on values of 0 to $100 \mathrm{ps}$ but also up to a few nanoseconds in loops, termini, and some side chains. Thus, ${ }^{15} \mathrm{~N}$ relaxation provides information on both the amplitude $\left(1-S^{2}\right)$ and timescale $\left(\tau_{\mathrm{e}}\right)$ for bond motion. Relaxation data for some residues fit better to model-free equations with different parameters besides the standard $S^{2}$ and $\tau_{\mathrm{e}}$ pair. In total, there are five model-free models [10], and statistical criteria[12] built into most fitting packages aid the user in choosing which model best suits the data for each residue. For some residues, elevated $R_{2}$ values dictate that data should be fit to models containing a $R_{e x}$ term which indicates motion on the $\mu \mathrm{s}-\mathrm{ms}$ timescale. It is important to note that the pulse sequence for measuring $R_{2}$ rates contains an element designed to suppress the effects of exchange on the ms time scale. Thus the absence of elevated $R_{2}$ rates does not necessarily rule out chemical exchange. In addition, experimenters should use caution in interpreting elevated $R_{2}$ values, as anisotropic tumbling can cause the same effect in the absence of chemical exchange. This lesson was exemplified with the case of MDM2, a p53 binding protein with an $\mathrm{N}$-terminal tail that is invisible in crystal structures. This tail was originally proposed to undergo chemical exchange based on an isotropic model of tumbling, but correct treatment of motional anisotropy changed the interpretation[13]*. Most model-free fitting packages guide the user through selecting the correct tumbling model. If the goal is to immediately discriminate between exchange and anisotropy, the $R_{1} R_{2}$ product is very sensitive to the former, but not the latter[14].

\section{NMR relaxation methods for characterizing ps-ns dynamics of side chain methyl groups}

Measuring dynamics at side-chain methyl groups is attractive because they are distributed throughout proteins and enriched within protein cores and binding interfaces. In addition, while backbone order parameters largely track with secondary structure (secondary structural elements are rigid, loops are flexible), the dynamic range of methyl order parameters is larger than the backbone counterparts (Figure 2) and are less predictable based on structure[5,15]. Values of methyl order parameters of 0.7 or less suggest the presence of rotameric switching in $\chi_{1}$ or $\chi_{2}[16,17]$. For methyl groups, order parameters are typically obtained from ${ }^{2} \mathrm{H}$ spin relaxation. Briefly, proteins are expressed in media supplemented with ${ }^{13} \mathrm{C}$-glucose, and $\sim 50 \% \mathrm{D}_{2} \mathrm{O}$ such that methyl groups are a mixture of isotopomers (i.e. ${ }^{13} \mathrm{CH}_{2} \mathrm{D}$, and ${ }^{13} \mathrm{CHD}_{2}$, where $\mathrm{D}$ is deuterium). Pulse sequences $[18,19]$ select for signals originating from ${ }^{13} \mathrm{CH}_{2} \mathrm{D}$ isotopomers and the degree of ${ }^{2} \mathrm{H}$ relaxation is then encoded in the intensity of ${ }^{1} \mathrm{H}_{-}{ }^{13} \mathrm{C}$ HSQC resonances (Figure 1). Typically, two experiments are performed, one measuring ${ }^{2} \mathrm{H} R_{1}$ and the other, ${ }^{2} \mathrm{H} R_{1 \rho}$ (similar to $R_{2}$ but with no contribution from chemical exchange). It is important to note that some versions of the pulse sequences measure three spin coherences, $\mathrm{I}_{\mathrm{z}} \mathrm{C}_{\mathrm{z}} \mathrm{D}_{\mathrm{z}}$ and $\mathrm{I}_{\mathrm{z}} \mathrm{C}_{\mathrm{z}} \mathrm{D}_{\mathrm{y}}$ (where $\mathrm{I}={ }^{1} \mathrm{H}, \mathrm{C}={ }^{13} \mathrm{C}$, and $\mathrm{D}={ }^{2} \mathrm{H}$ ). Thus, in order to obtain pure ${ }^{2} \mathrm{H}$ relaxation rates, a third experiment, $\mathrm{I}_{\mathrm{Z}} \mathrm{C}_{\mathrm{Z}}$, must be run and subtracted from the other two. These data are then fit to the model-free formalism. The order parameters correspond to $\mathrm{C}-\mathrm{CH}_{3}$ bond vectors and are referred to as $S^{2}$ axis, where 'axis' refers to the 3-fold symmetry axis collinear with the $\mathrm{C}-\mathrm{CH}_{3}$ bond and $S^{2}$ axis $=S^{2} \mathrm{CH}_{3} / 0.111$. The sensitivity of these experiments degrades rapidly as the molecular weight of the protein increases beyond $30 \mathrm{kDa}$. Recent developments in assignment strategies, isotopic labeling schemes, and pulse sequence design have increased this size limit to over $100 \mathrm{kDa}$ for some favorable cases[20]*. 


\section{Nature uses ps-ns fluctuations to modulate protein function}

There is growing evidence that all proteins employ dynamics for function. While $\mu$ s-ms motions are often emphasized for being commensurate with the timescales of binding and catalysis, ps-ns motions are universal (not all proteins exhibit $\mu$ s-ms motions) and faster fluctuations have been proposed to facilitate exchange events on slower timescales[21]. The following is a sampling of cases where the linkage between dynamics on the ps-ns time scale and function is especially dramatic. Prior to the advent of NMR relaxation methods, protein flexibility was often inferred from x-ray crystal structures. For example, the structure of free calmodulin showed that the two lobes which must come together to envelope target peptides are separated by a 26 -residue $\alpha$-helix. In one of the first implementations of the model-free formalism, Bax and coworkers were able to reconcile this paradox and show that the middle of central "helix" is flexible in solution on the timescale of hundreds of picoseconds[22]. This central kink in the helix allows the two lobes to come together and bind targets. As another example, crystal structures of the free HIV protease implied the necessity for flexibility because the active sites were occluded by $\beta$-hairpins. Indeed, order parameters revealed that these $\beta$-hairpin "flaps" are flexible on the ps-ns timescale[23], with the fluctuations functioning to allow substrate access to and release of products from the active site. It was also shown that a high degree of ps-ns flexibility is advantageous to DNA binding proteins $[24,25]$ as they must locate their target sites by navigating a vast array of non specific sites while sliding along the double helix. Lastly, fast fluctuations can modulate specificity. This is best exemplified by non-NMR studies of antibodies[26,27] showing that ps-ns flexibility is important for the broad specificity early on in affinity maturation and a stepwise decrease in flexibility of the binding site accompanies the stepwise increase in affinity for the target. NMR studies showed dynamics on this timescale influence specificity in a number of other systems[28,29].

\section{Order parameters as a proxy for conformational entropy: Calmodulin as a model system}

Structure-based drug design is employed because the relative binding affinities of a series of lead compounds can often be predicted based on the coordinates of static structural models. One of the ways NMR dynamics measurements can be of aid to the drug design community is if order parameters represent a window into molecular driving forces that are invisible to traditional structure based tools. Indeed, it was demonstrated almost two decades ago, that atomic coordinate fluctuation as measured by $S^{2}$, are related to the canonical partition function, and hence the Gibbs free energy[30]. Soon thereafter, analytical expressions equating NMR order parameters to absolute conformational entropy were derived[31,32]. While the absolute entropies were highly dependent on the models used to describe the motion, relative entropies (i.e. between free and bound states) were robust and model independent. It was therefore predicted that differences in order parameters could be a proxy for differences in conformational entropy given the assumption that there are no correlated motions or the extent of correlated motions is the same in both states.

It was not until recently that Wand and coworkers performed the systematic study required to test this idea[33]**. Calorimetric measurements on calmodulin $(\mathrm{CaM})$ binding to a series of six target peptides showed there is a large range in overall $\mathrm{T} \Delta \mathrm{S}$ for binding $(\sim 22 \mathrm{kcal}$ $\left.\mathrm{mol}^{-1}\right)$ despite the relatively constant overall $\Delta \mathrm{G}\left(\sim 3 \mathrm{kcal} \mathrm{mol}^{-1}\right)[34,35]$. This large "dynamic range" in the overall entropy change, together with the fact that all six peptides are amphiphilic $\alpha$-helices which bind to the same site on CaM made the system ideal. Sidechain order parameters were measured for free calmodulin $(\mathrm{CaM})$ and $\mathrm{CaM}$ bound to the set of target peptides, and were subsequently used to calculate the conformational entropy changes for the binding reactions. The calculated entropies were compared to the total 
calorimetric entropies, and a linear correlation between the two datasets was observed[33]. This provided the first experimental evidence that NMR order parameters are a proxy for entropy and are therefore reporters on a significant contributor to binding free energy.

While the linear relationship between the conformational entropy change derived from NMR order parameters $\left(\Delta \mathrm{S}_{\text {conf }}^{\mathrm{CaM}}\right)$ and total entropy change $\left(\Delta \mathrm{S}_{\text {tot }}\right)$ was highly suggestive, the quantitative contribution of $\Delta S_{\text {conf }}^{\mathrm{CaM}}$ remained unknown due to the potential variability in the other factors influencing the total $\Delta \mathrm{S}$ (equation 1)

$$
\Delta \mathrm{S}_{\mathrm{tot}}=\Delta \mathrm{S}_{\mathrm{conf}}^{\mathrm{CaM}}+\Delta \mathrm{S}_{\mathrm{conf}}^{\mathrm{target}}+\Delta \mathrm{S}_{\mathrm{sol}}+\Delta \mathrm{S}_{\mathrm{RT}}
$$

In equation 1: $\Delta S_{\text {tot }}$ represents the calorimetric entropy, the conformational entropy change is further divided into contributions from $\mathrm{CaM}$ and its target peptide, $\Delta \mathrm{S}_{\text {sol }}$ represents changes in solvent entropy, and $\Delta \mathrm{S}_{\mathrm{RT}}$ represents entropy change due to loss of translational and rotational degrees of freedom upon binding. Based on this decomposition of the total entropy, Wand and coworkers reasoned that it would be possible to calibrate a "conformational entropy meter" if the changes in dynamics of the target peptide and changes in $\Delta \mathrm{S}_{\mathrm{sol}}$ are taken into account[36]*. In this study, Marlow et al. used the same series of six CaM-target pairs, measured the side-chain dynamics from the target peptide to access $\Delta \mathrm{S}_{\text {conf }}^{\text {target }}$, and calculated $\Delta \mathrm{S}_{\text {sol }}$ from crystal structures using accessible surface area (ASA) approaches. From this they observed the following linear relationship (equation 2):

$$
\left(\Delta \mathrm{S}_{\mathrm{tot}}-\Delta \mathrm{S}_{\mathrm{sol}}\right)=m\left[\left(n_{\mathrm{res}}^{\mathrm{CaM}} \bullet\left\langle\Delta S_{\text {axis }}^{2}\right\rangle^{\mathrm{CaM}}+n_{\mathrm{res}}^{\mathrm{target}} \bullet\left\langle\Delta S_{\text {axis }}^{2}\right\rangle^{\text {target }}\right)\right]+\Delta \mathrm{S}_{\mathrm{RT}}+\Delta \mathrm{S}_{\mathrm{other}}
$$

(Note that order parameter " $\mathrm{S}^{2}$ " is italicized to distinguish it from entropy. Marlow et al. substituted with " $\mathrm{O}^{2}$ " for this reason.) Linearity of equation 2 demonstrates the following: 1) $\Delta \mathrm{S}_{\text {conf }}$ is represented by the slope times the abscissa coordinate. 2) By scaling the average change in order parameter $\left\langle\Delta S_{\text {axis }}^{2}\right\rangle$, by the number of residues in the calculation ( $n_{\text {res }}$ terms), binding partners with different numbers of NMR probes (assuming the ratio of probes used to total number of residues is relatively constant in the series) can be used to generate the model or as input for the "entropy meter". 3) The y-intercept reports on $\Delta \mathrm{S}_{\mathrm{RT}}+\Delta \mathrm{S}_{\mathrm{other}}$, which is the same for each complex. 4) Variability in $\Delta S_{\text {tot }}$ can be completely accounted for by changes in methyl order parameters and ASA. This study showed that $\Delta \mathrm{S}_{\text {conf }}$ and $\Delta \mathrm{S}_{\text {sol }}$ were of roughly equal magnitude and opposite sign with both of these parameters being greater than the overall entropy change for binding. Therefore conformational entropy can be a true driving force in binding processes with the overall entropy change governed by the balance of the large and opposing solvation and conformational terms. It remains an open question as to whether this type of predictive power can be gleaned from similar studies on other homologous series and how related the binding reactions within a series must be for a linear relationship to be observed. We restate the caveat that accuracy of order parameter based methods for calculating differences in entropies requires that coupled motions are either absent or similar in both states. While success of the entropy meter approach shows this to be a reasonable assumption for calmodulin, recent molecular dynamics based calculations of absolute entropies in biomolecules show the effects of coupled motions can be large[37,38].

Finally, an alternative method for translating order parameters into conformational entropy draws upon molecular dynamics (MD) simulations. Because both order parameters and conformational entropy can be directly computed from MD simulations, $\mathrm{Li}$ and 
Brüschweiler developed empirical simple scaling between the two for different classes of bond vectors[39]. The correspondence between $S^{2}$ and conformational entropy was remarkably linear. In principle, this allows for simple conversion of experimental $S^{2}$ values into conformational entropies.

\section{ps-ns dynamics in the form of entropy can drive allostery}

Targeting multiple proteins with different drugs has proven an effective strategy for battling resistance in HIV. An alternative is to target multiple sites on the same protein. Recently, it was shown that a pair of drugs, one binding to the ATP site, and the other binding to an allosteric site on Bcr-Abl is effective in overcoming drug resistant forms of chronic myeloid leukemia[40,41]. The promise of other drug targets with multiple, communicating sites provides the motivation to more deeply understand the rules governing allostery. Traditional models of allostery rely on conformational change to explain the phenomenon. However, it was proposed nearly three decades ago that it should be possible for allostery to be manifested by purely entropic means, that is, without net conformational change[42]. In this model, perturbation (i.e. mutation or binding) at one site affects the free energy change for a downstream event by influencing macromolecular fluctuations. Recently, NMR spin relaxation studies have revealed that entropically (dynamically) driven allostery is a bona fide mechanism utilized by nature. One example involves the catabolite activator protein (CAP), a dimeric transcription factor that binds two cAMP molecules with strong negative cooperativity. Popovych et al. used NMR probes of structure and dynamics to understand cooperativity in the N-terminal, cAMP binding domain (CBD) of CAP. Interestingly, binding of the first cAMP did not cause structural changes in the second binding site[43]*, leaving the possibility that dynamics are responsible for allostery. Backbone order parameters were then measured from apo- $\mathrm{CBD}_{2}, \mathrm{cAMP}-\mathrm{CBD}_{2}$, and $\mathrm{cAMP} \mathrm{ABD}_{2}-\mathrm{CBD}_{2}$. These showed that binding of the first cAMP molecule caused very little change in the ps-ns dynamics of either subunit, while binding of the second molecule induced widespread quenching of dynamics (and loss of conformational entropy) in both subunits. The inferred conformational entropy mechanism of negative cooperativity was shown to be consistent with the overall entropies of binding measured by ITC. A second example involves a mutant in the cAMP binding domain of full length CAP. The S62F CAP variant binds to DNA with the exact same affinity as the wild-type, yet there is $27.5 \mathrm{kcal} / \mathrm{mol}$ difference in $T \Delta S$ between the two binding interactions[44]. Differences in backbone NMR order parameters between the $\mathrm{CAMP}_{2}-\mathrm{CAP}_{2}$ and $\mathrm{cAMP}_{2}-\mathrm{CAP}_{2}$-DNA states explained this observation in that the cAMP domain of the mutant was much more flexible than the wild-type in the DNA-bound state.

A final example of dynamically driven allostery comes from our lab studying the interaction between the third PDZ domain (PDZ3) from post-synaptic density 95 (PSD-95) and a Cterminal peptide from the CRIPT protein. PDZ3 has a C-terminal $\alpha$-helix that is not present in most PDZ domains. Interestingly, this helix is phosphorylated at a location that may interfere with packing against the rest of the PDZ domain. To interrogate the role of a Cterminal helix in CRIPT binding we made a helical deletion mutant $(\Delta 7 \mathrm{ct})$ and found $\Delta 7 \mathrm{ct}$ binds to the CRIPT peptide 20-fold more weakly than the wild-type despite the fact that the $\mathrm{C}$-terminal helix does not contact the CRIPT peptide[45]*. The measured $\Delta \mathrm{H}$ values for wild-type and $\Delta 7 \mathrm{ct}$ binding are exactly the same so the difference in binding is entirely entropic. Further, changes in NMR chemical shifts between free and bound deletion mutants were the same as those from the wild-type counterparts, effectively eliminating conformational change as a mechanism for the allosterically induced binding penalty. Instead, ${ }^{2} \mathrm{H}$ relaxation studies show that truncation results in a global increase in the flexibility of methyl side chains in the unbound state such that the unfavorable change in conformational entropy upon peptide binding is increased relative to the wild-type. 


\section{Combining NMR relaxation and molecular dynamics tools overcomes the limitations of each}

Biomolecules exist as ensembles of structures, and function is often carried out by lowly populated conformations that are not represented by crystal structures. For such cases, docking of rigid drug candidates to a rigid receptor will yield incorrect binding poses and misleading scores[46]. There are now many ways to incorporate flexibility into docking algorithms but serious limitations such as inadequate sampling of conformational space and bias towards the experimental structural model remain[47]. On the other side of the coin, the strength of NMR ps-ns relaxation methods lies in the fact that population weighted dynamics of the entire solution ensemble are extracted, yet order parameters do not give physical descriptions of the motions. Dobson, Vendruscolo, and colleagues recognized that the shortcomings of the simulation and NMR tools mentioned above could be eliminated if both approaches were combined. In the method termed dynamic ensemble refinement [48]* the CHARMM molecular mechanics force-field, NOE distances, and NMR order parameters (both backbone and side-chain) were used as restraints (equation 3) in generating an ensemble of ubiquitin structures.

$$
E_{\mathrm{tot}}=E_{\mathrm{CHARM}}+E_{\mathrm{NOE}}+E_{S^{2}}
$$

The ensemble was cross-validated against a large series of NMR residual dipolar coupling (RDC) measurements and was found to more faithfully represent these solution data than either the crystal structure or the NMR ensemble. This underscored the importance of the order parameters in defining the envelope of solution conformations. Further, because RDCs report on processes that occur on timescales from ns-ms, the ensemble encompasses conformational space that is inaccessible to short molecular dynamics simulations. This was a powerful demonstration of the complementary nature of modern NMR and computational tools.

\section{Conclusions}

Up until the first decade of the $21^{\text {st }}$ century, with the exception of a minority of structural biologists, proteins were viewed as static structures. This view is undergoing a vast transformation throughout the biological sciences to one in which proteins are understood to dynamically sample a myriad of substructures within, or even outside, the overall fold. Our appreciation for this has likely been aided by the recognition that many functional proteins are intrinsically disordered. NMR spectroscopy has been instrumental in recording experimental evidence for protein dynamism. Protein motions occur on timescales from ps to days, and NMR methods are sensitive to essentially this entire timescale. Nevertheless, motions on the ps-ns timescale are particularly amenable to quantitative characterization of amplitudes of motion, through the "model-free" order parameter, $S^{2}$. These "fast" order parameters are obtained from spin relaxation measurements (e.g., $\left.{ }^{15} \mathrm{~N},{ }^{13} \mathrm{C},{ }^{2} \mathrm{H}\right)$ and capture motion that may have a high degree of diffusive motion. Motions on slower timescales are not easily characterized by order parameters, yet those motions may be more concerted and switch-like. In terms of protein function and drug design, fast backbone and side-chain motions appear to have their largest influence through conformational entropy, as discussed in this review. However, correlating fast dynamics (i.e., $S^{2}$ ) with function in other ways is often not straightforward, even though vital connections likely exist. This difficulty may stem from the relatively simple information contained in $S^{2}$. Future progress for correlating fast dynamics with function is likely to depend on combining NMR and MD simulations, as well as investigators' ingenuity in experimental design to probe these relationships. 


\section{References}

*1. Mobley DL, Dill KA. Binding of small-molecule ligands to proteins: "what you see" is not always "what you get". Structure 2009;17:489-498. [PubMed: 19368882] This insightful review outlines how single static structures do not reveal all the important forces in macromolecular binding. Computational strategies for quantifying these invisible forces are described.

2. Palmer AG 3rd, Kroenke CD, Loria JP. Nuclear magnetic resonance methods for quantifying microsecond-to-millisecond motions in biological macromolecules. Methods Enzymol 2001;339:204-238. [PubMed: 11462813]

3. Mittermaier A, Kay LE. New tools provide new insights in NMR studies of protein dynamics. Science 2006;312:224-228. [PubMed: 16614210]

**4. Jarymowycz VA, Stone MJ. Fast time scale dynamics of protein backbones: NMR relaxation methods, applications, and functional consequences. Chem Rev 2006;106:1624-1671. [PubMed: 16683748] A thorough review of theoretical and practical aspects of NMR methods used to characterize ps-ns dynamics of the protein backbone.

**5. Igumenova TI, Frederick KK, Wand AJ. Characterization of the fast dynamics of protein amino acid side chains using NMR relaxation in solution. Chem Rev 2006;106:1672-1699. [PubMed: 16683749] A thorough review of theoretical and practical aspects of NMR methods used to characterize ps-ns dynamics of the protein side-chain methyl groups.

6. John Cavanagh, WJF.; Pamer, Arthur G., III; Rance, Mark; Skelton, Nicholas J. Protein NMR Spectroscopy: Principles and Practice. edn 2. Eslevier; 2007.

7. Lipari G, Szabo A. Model-Free Approach to the Interpretation of Nuclear Magnetic-Resonance Relaxation in Macromolecules 1. Theory and Range of Validity. Journal of the American Chemical Society 1982;104:4546-4559.

8. Lipari G, Szabo A. Model-Free Approach to the Interpretation of Nuclear Magnetic-Resonance Relaxation in Macromolecules 2. Analysis of Experimental Results. Journal of the American Chemical Society 1982;104:4559-4570.

9. Farrow NA, Muhandiram R, Singer AU, Pascal SM, Kay CM, Gish G, Shoelson SE, Pawson T, Forman Kay JD, Kay LE. Backbone Dynamics of a Free and a Phosphopeptide-Complexed Src Homology-2 Domain Studied by N-15 NMR Relaxation. Biochemistry 1994;33:5984-6003. [PubMed: 7514039]

10. Mandel AM, Akke M, Palmer AG 3rd. Backbone dynamics of Escherichia coli ribonuclease HI: correlations with structure and function in an active enzyme. J Mol Biol 1995;246:144-163. [PubMed: 7531772]

11. Dosset P, Hus JC, Blackledge M, Marion D. Efficient analysis of macromolecular rotational diffusion from heteronuclear relaxation data. J Biomol NMR 2000;16:23-28. [PubMed: 10718609]

12. d'Auvergne EJ, Gooley PR. The use of model selection in the model-free analysis of protein dynamics. J Biomol NMR 2003;25:25-39. [PubMed: 12566997]

*13. Showalter SA, Bruschweiler-Li L, Johnson E, Zhang F, Bruschweiler R. Quantitative lid dynamics of MDM2 reveals differential ligand binding modes of the p53-binding cleft. J Am Chem Soc 2008;130:6472-6478. [PubMed: 18435534] In this study, NMR order parameters reveal that ps-ns fluctuations of the oncoprotein MDM2 backbone influence the binding of a p53derived peptide. The paper also presents an important case study in how properly accounting for anisotropic tumbling is critical for interpretation of NMR relaxation data.

14. Kneller JM, Lu M, Bracken C. An effective method for the discrimination of motional anisotropy and chemical exchange. J Am Chem Soc 2002;124:1852-1853. [PubMed: 11866588]

15. Ming D, Bruschweiler R. Prediction of methyl-side chain dynamics in proteins. J Biomol NMR 2004;29:363-368. [PubMed: 15213434]

16. Chou JJ, Case DA, Bax A. Insights into the mobility of methyl-bearing side chains in proteins from (3) J(CC) and (3)J(CN) couplings. J Am Chem Soc 2003;125:8959-8966. [PubMed: 12862493]

17. Hu H, Hermans J, Lee AL. Relating side-chain mobility in proteins to rotameric transitions: insights from molecular dynamics simulations and NMR. J Biomol NMR 2005;32:151-162. [PubMed: 16034666] 
18. Muhandiram DR, Yamazaki T, Sykes BD, Kay LE. Measurement of H-2 T-1 and T-1p RelaxationTimes in Uniformly C-13-Labeled and Fractionally H-2-Labeled Proteins in Solution. Journal of the American Chemical Society 1995;117:11536-11544.

19. Millet O, Muhandiram DR, Skrynnikov NR, Kay LE. Deuterium spin probes of side-chain dynamics in proteins. 1. Measurement of five relaxation rates per deuteron in (13)C-labeled and fractionally (2)H-enriched proteins in solution. J Am Chem Soc 2002;124:6439-6448. [PubMed: 12033875]

*20. Sheppard D, Sprangers R, Tugarinov V. Experimental approaches for NMR studies of side-chain dynamics in high-molecular-weight proteins. Progress in Nuclear Magnetic Resonance Spectroscopy 2010;56:1-45. [PubMed: 20633347] A review describing advances in isotopic labeling schemes, resonance assignment methods, and pulse sequence design that have allowed for NMR dynamic characterization of large proteins and supramolecular assemblies approaching one megadalton.

21. Henzler-Wildman KA, Lei M, Thai V, Kerns SJ, Karplus M, Kern D. A hierarchy of timescales in protein dynamics is linked to enzyme catalysis. Nature 2007;450:913-916. [PubMed: 18026087]

22. Barbato G, Ikura M, Kay LE, Pastor RW, Bax A. Backbone dynamics of calmodulin studied by $15 \mathrm{~N}$ relaxation using inverse detected two-dimensional NMR spectroscopy: the central helix is flexible. Biochemistry 1992;31:5269-5278. [PubMed: 1606151]

23. Freedberg DI, Ishima R, Jacob J, Wang YX, Kustanovich I, Louis JM, Torchia DA. Rapid structural fluctuations of the free HIV protease flaps in solution: relationship to crystal structures and comparison with predictions of dynamics calculations. Protein Sci 2002;11:221-232. [PubMed: 11790832]

24. Kalodimos CG, Biris N, Bonvin AM, Levandoski MM, Guennuegues M, Boelens R, Kaptein R. Structure and flexibility adaptation in nonspecific and specific protein-DNA complexes. Science 2004;305:386-389. [PubMed: 15256668]

25. Lee GM, Donaldson LW, Pufall MA, Kang HS, Pot I, Graves BJ, McIntosh LP. The structural and dynamic basis of Ets-1 DNA binding autoinhibition. J Biol Chem 2005;280:7088-7099. [PubMed: 15591056]

26. Thorpe IF, Brooks CL 3rd. Molecular evolution of affinity and flexibility in the immune system. Proc Natl Acad Sci U S A 2007;104:8821-8826. [PubMed: 17488816]

27. Zimmermann J, Oakman EL, Thorpe IF, Shi X, Abbyad P, Brooks CL 3rd, Boxer SG, Romesberg FE. Antibody evolution constrains conformational heterogeneity by tailoring protein dynamics. Proc Natl Acad Sci U S A 2006;103:13722-13727. [PubMed: 16954202]

28. Das R, Chowdhury S, Mazhab-Jafari MT, Sildas S, Selvaratnam R, Melacini G. Dynamically driven ligand selectivity in cyclic nucleotide binding domains. J Biol Chem 2009;284:2368223696. [PubMed: 19403523]

29. Peng T, Zintsmaster JS, Namanja AT, Peng JW. Sequence-specific dynamics modulate recognition specificity in WW domains. Nat Struct Mol Biol 2007;14:325-331. [PubMed: 17334375]

30. Akke M, Bruschweiler R, Palmer AG. Nmr Order Parameters and Free-Energy - an Analytical Approach and Its Application to Cooperative Ca2+ Binding by Calbindin-D(9k). Journal of the American Chemical Society 1993;115:9832-9833.

31. Li Z, Raychaudhuri S, Wand AJ. Insights into the local residual entropy of proteins provided by NMR relaxation. Protein Sci 1996;5:2647-2650. [PubMed: 8976574]

32. Yang D, Kay LE. Contributions to conformational entropy arising from bond vector fluctuations measured from NMR-derived order parameters: application to protein folding. J Mol Biol 1996;263:369-382. [PubMed: 8913313]

**33. Frederick KK, Marlow MS, Valentine KG, Wand AJ. Conformational entropy in molecular recognition by proteins. Nature 2007;448:325-U323. [PubMed: 17637663] This study provides the first real evidence that changes in side-chain methyl order parameters are a proxy for a sizable contributor to the overall entropy change and hence the free energy change for binding.

34. Frederick KK, Kranz JK, Wand AJ. Characterization of the backbone and side chain dynamics of the CaM-CaMKIp complex reveals microscopic contributions to protein conformational entropy. Biochemistry 2006;45:9841-9848. [PubMed: 16893184] 
35. Marlow MS, Wand AJ. Conformational dynamics of calmodulin in complex with the calmodulindependent kinase kinase alpha calmodulin-binding domain. Biochemistry 2006;45:8732-8741. [PubMed: 16846216]

*36. Marlow MS, Dogan J, Frederick KK, Valentine KG, Wand AJ. The role of conformational entropy in molecular recognition by calmodulin. Nat Chem Biol 2010;6:352-358. [PubMed: 20383153] This study successfully calibrates an "entropy meter" that can estimate of the magnitude of the conformational entropy change upon calmodulin binding to a homologous series of target peptides. Conformational entropy changes are found to be equal in magnitude and opposite in sign as compared to the more widely appreciated solvent release contribution.

37. Baron R, Hunenberger PH, McCammon JA. Absolute Single-Molecule Entropies from QuasiHarmonic Analysis of Microsecond Molecular Dynamics: Correction Terms and Convergence Properties. J Chem Theory Comput 2009;5:3150-3160. [PubMed: 20011626]

38. Baron R, McCammon JA. (Thermo)dynamic role of receptor flexibility, entropy, and motional correlation in protein-ligand binding. Chemphyschem 2008;9:983-988. [PubMed: 18418822]

39. Li DW, Bruschweiler R. A dictionary for protein side-chain entropies from NMR order parameters. J Am Chem Soc 2009;131:7226-7227. [PubMed: 19422234]

40. Zhang J, Adrian FJ, Jahnke W, Cowan-Jacob SW, Li AG, Iacob RE, Sim T, Powers J, Dierks C, Sun F, et al. Targeting Bcr-Abl by combining allosteric with ATP-binding-site inhibitors. Nature 2010;463:501-506. [PubMed: 20072125]

41. Weisberg E, Deng X, Choi HG, Barrett R, Adamia S, Ray A, Moreno D, Kung AL, Gray N, Griffin JD. Beneficial effects of combining a type II ATP competitive inhibitor with an allosteric competitive inhibitor of BCR-ABL for the treatment of imatinib-sensitive and imatinib-resistant CML. Leukemia 2010;24:1375-1378. [PubMed: 20508612]

42. Cooper A, Dryden DT. Allostery without conformational change. A plausible model. Eur Biophys J 1984;11:103-109. [PubMed: 6544679]

*43. Popovych N, Sun S, Ebright RH, Kalodimos CG. Dynamically driven protein allostery. Nat Struct Mol Biol 2006;13:831-838. [PubMed: 16906160] This study uses multiple NMR tools to provide the first experimental evidence that allostery can occur without conformational change. Order parameters show that binding of the first effector molecule to this dimeric DNA binding protein makes binding of the second less favorable based on a dynamically driven (entropic) mechanism. The idea was proposed to be possible in theory two decades earlier (see [42]).

44. Tzeng SR, Kalodimos CG. Dynamic activation of an allosteric regulatory protein. Nature 2009;462:368-372. [PubMed: 19924217]

*45. Petit CM, Zhang J, Sapienza PJ, Fuentes EJ, Lee AL. Hidden dynamic allostery in a PDZ domain. Proc Natl Acad Sci U S A 2009;106:18249-18254. [PubMed: 19828436] By measuring sidechain methyl order parameters, we demonstrate that removal of a PDZ3 $\alpha$-helix that is distal to the peptide binding site modulates binding affinity through a conformational entropy mechanism.

46. Totrov M, Abagyan R. Flexible ligand docking to multiple receptor conformations: a practical alternative. Curr Opin Struct Biol 2008;18:178-184. [PubMed: 18302984]

47. B-Rao C, Subramanian J, Sharma SD. Managing protein flexibility in docking and its applications. Drug Discov Today 2009;14:394-400. [PubMed: 19185058]

*48. Lindorff-Larsen K, Best RB, Depristo MA, Dobson CM, Vendruscolo M. Simultaneous determination of protein structure and dynamics. Nature 2005;433:128-132. [PubMed: 15650731] By using a structural refinement strategy that incorporates both NMR order parameters and a molecular dynamics force-field, an ensemble of ubiquitin structures is generated that accurately represents conformational spread in solution. The study demonstrates that by combining NMR relaxation and computational tools, the shortcomings of each can be masked and the respective strengths emphasized. 


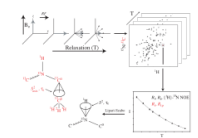

Figure 1.

Basic flow-chart for characterization of ps-ns dynamics by NMR. A pictorial representation of $T_{2}$ relaxation is presented in the first quadrant. The presence of a static magnetic field $\left(B_{0}\right)$ results in a bulk magnetization (grey arrow) aligned with $B_{0}$. An RF pulse rotates the bulk magnetization into the $x y$ plane. The individual spins de-phase during the relaxation time (T), leading to the exponential decrease in the bulk magnetization as a function of $\mathrm{T}$. The rate of loss of spin coherence is referred to as the spin-spin relaxation rate $\left(R_{2}\right)$. Molecular tumbling and internal fluctuations affect this rate of relaxation. Relaxation is monitored by acquisition of 2D HSQC type spectra with variable times of $\mathrm{T}$ (upper right quadrant). The spectra are ${ }^{1} \mathrm{H}_{-}{ }^{15} \mathrm{~N}$ and ${ }^{1} \mathrm{H}-{ }^{13} \mathrm{C}$ for ${ }^{15} \mathrm{~N}$ and ${ }^{2} \mathrm{H}$ relaxation, respectively. ${ }^{15} \mathrm{~N}$ relaxation typically involves measurement of two rates, $R_{1}$ and $R_{2}$, and the $\left\{{ }^{1} \mathrm{H}\right\}-{ }^{15} \mathrm{~N}$ heteronuclear NOE; ${ }^{2} \mathrm{H}$ relaxation involves measurement of $R_{1}$ and $R_{1 \mathrm{p}}$. To obtain relaxation rates, the decays in resonance intensities are fit to single exponential equations (lower right quadrant). These raw relaxation parameters are subsequently fit to Lipari-Szabo model-free equations in order to obtain parameters describing amplitude (the order parameter, $S^{2}$ ), and characteristic time $\left(\tau_{\mathrm{e}}\right)$ of bond vector motion (lower left quadrant). Note that the model-free parameters report on the amide ${ }^{1} \mathrm{H}-{ }^{15} \mathrm{~N}$ bond vector (black) and the $\mathrm{C}-\mathrm{CH}_{3}$ symmetry axis (alanine example in red) for ${ }^{15} \mathrm{~N}$ and ${ }^{2} \mathrm{H}$ relaxation, respectively. 


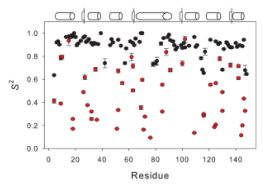

Figure 2.

Side-chain flexibility is more heterogeneously distributed than main-chain flexibility. Backbone amide $S^{2}$ values (black) and side-chain methyl $S^{2}$ axis values (red) for $\mathrm{Ca}^{2+}$-bound calmodulin are plotted. Note that the backbone values are tightly clustered around 0.9 with the exceptions of loops and the central helix ( $\alpha$-helices and $\beta$-sheets are depicted by cylinders and arrows respectively at the top of the figure). The side-chain methyl order parameters are more heterogeneous and have no relation to the protein secondary structure. Data were obtained from the Biological Magnetic Resonance Data Bank (accession number 15188). 